\title{
Organotin Compounds in Sediments of Northern Lakes, Egypt
}

\author{
Mohamed A. Shreadah1* ${ }^{*}$, Safaa A. Abdel Ghani1, Hamada B. I. Hawash1, Asia Abd El Samie², \\ Abd El Moniem M. Ahmed ${ }^{2}$ \\ ${ }^{1}$ National Institute of Oceanography and Fisheries, Alexandria, Egypt \\ ${ }^{2}$ Chemistry Department, Faculty of Science, Alexandria University, Alexandria, Egypt \\ Email: ${ }^{\text {niof.shreadah@gmail.com }}$
}

Received 14 October 2014; revised 12 November 2014; accepted 7 December 2014

Copyright (C) 2014 by authors and Scientific Research Publishing Inc.

This work is licensed under the Creative Commons Attribution International License (CC BY). http://creativecommons.org/licenses/by/4.0/

(c) (i) Open Access

\section{Abstract}

This pioneered study is aimed to determine the levels and distributions of organotin compounds (OTCs) in sediment samples collected from five Egyptian lakes located in the southern-eastern Mediterranean Sea. The paper has given an account of and the reasons for the widespread distribution of Tributyltin (TBT) in northern lakes. The percentage of TBT ranged from $4 \%-100 \% 0 T C$ revealing recent inputs of TBT. The highest average of TBT $(2.84 \mu \mathrm{g} / \mathrm{g} \mathrm{Sn} \mathrm{dry} \mathrm{wt)} \mathrm{was} \mathrm{measured} \mathrm{in}$ lake Edku. Dibutyltin (DBT) was observed at most sampled sites. Relatively higher abundance of DBT was observed in sediments of EL-Burullus $(0.69 \mu \mathrm{g} / \mathrm{g}$ Sn dry wt). One of most significant findings to emerge from this study is the absence of any strong and significant correlation between TBT and DBT in sediments from five northern lakes. The absence of such correlation might give a clear evidence that both compounds did not come from the same source. The observed OTC levels indicate some highly localized areas of contamination which are severe enough to cause harmful effects on marine flora and fauna. Except for El-Burllus, Triphenyltin (TPhT) was not measured in sediments of most stations. TPhT was the predominant species in El-Burullus, which indicate a source of pollution mostly originated from industrial and agricultural waste water discharge, organic wastes, commercial fertilizers, chemical wastes and pesticides. The percentages of OTC to total tin ranged from 1\% to 35\%. El-Brullus, Edku and El-Bardaweel exhibited the highest percentages. As a result of lack of enforcement on the control of OTC, the study has thrown up many questions need further detailed environmental assessment of OTC.

\section{Keywords}

Organotin Compounds, Sediments, Lakes, Egypt

\footnotetext{
"Corresponding author.
}

How to cite this paper: Shreadah, M.A., Ghani, S.A.A., Hawash, H.B.I., El Samie, A.A. and Ahmed, A.E.M.M. (2014) Organotin Compounds in Sediments of Northern Lakes, Egypt. Journal of Environmental Protection, 5, 1654-1666. 


\section{Introduction}

Tin compounds are sparingly soluble in water and are likely to partition to soils and sediments. Most commercially used OTCs are relatively immobile in environment media due to their low vapor pressures, low water solubility and high affinity for soil and organic sediments [1]. Inorganic Sn cannot be degraded in the environment, but may undergo oxidation-reduction, ligand exchange and precipitation reactions [2]. Degradation of organotin compounds in sediments is much slower than in water, and half lives have been estimated to be several years [3].

In spite of legislative regulations for OTC, it is likely that OTC will continue to be produced and used as effective biocides, especially in developing countries. They continue to be used also in material and wood preservatives as effective biocides [4]. TBT has been considered the most hazardous compound to marine organisms and it is perhaps the most toxic chemical that has ever been introduced to marine environment [5] [6]. The great stability of the TBT adsorbed onto sediments shows the long-term storage capacity of this compartment [7]. It is well known that sediment is not a storage place but serves as a renewable source for OTC, especially TBT [8] [9]. Triphenyltin (TPhT) compounds metabolize to diphenyltin, mono-phenyltin and non-extractable bound residues.

DBT has mainly been related to the degradation of TBT via microbial activity and/or photochemical reactions. In recent years evidence for a direct entrance of DBT into the environment was found due to municipal wastewaters, sewage sludge and land fill leachates. For example, the major application of DBT is as additives to plastics particularly to polyvinyl chloride (PVC) to prevent degradation due to changes in temperature or lighting. PVC is used in water pipes rigid sheeting (e.g. for roofing) and bottles [10]. DBT compounds are characterized by higher water solubility than TBT, which probably gives rise to higher concentrations in groundwater and freshwater.

TPhT compounds have been used extensively as algaecide and molluscicides in antifouling products since 1960s [11]. TPhT is a co-toxicant in some long-term antifouling paints [12] and is similar with TBT in toxicity [13]. The persistence of the TPhT in soil is affected by a range of soil parameters including microbial activity and sequestration, moisture content and soil type affecting adsorption and hence bioavailability [14]. It is well known that TPhT is strongly adsorbed to sediments and soil, with no desorption [15]-[17]. TphT compounds are widely used in agriculture as fungicides. The use of TPhT has not been as strictly regulated as TBT but it is acknowledged to produce similar levels of toxicity [18].

Despite the presence of few studies that have been conducted to investigate the levels and distribution of OTC in the south eastern Mediterranean Sea [15] [19]-[21], this study is pioneered to determine the levels and distributions of OTC in sediments of Egyptian lakes.

The Egyptian Mediterranean coast exhibits five lakes (Figure 1), Mariout, Edku, El-Burullus, El-Manzallah and El-Bardaweel. All of them, with the exception of Lake Mariout are directly connected to the Mediterranean Sea through several straits. The northern lakes are economically the most important fishing ground. They are providing a rich and vital habitat for estuarine and marine fish and their regeneration. Northern lakes have always been major areas of fish production in the south eastern Mediterranean Sea, since more than $75 \%$ of the Egyptian lakes productions are harvesting from them. They are also important sites for wintering water birds providing valuable habitats for several hundred thousand of birds. Unfortunately many challenges are facing these lakes i.e. degradation and decreasing the lakes area in addition to pollution problems such as increasing the levels of heavy metals, pesticides, etc. in water, sediments and biota which are increasing due to the expansion of human activities, fish farming activities and aquatic plants.

The aim of this study is to determine the levels and distributions of organotin compounds (OTCs) in sediment samples collected from these Egyptian lakes located in the southern-eastern Mediterranean Sea.

\section{Materials and Methods}

Fifty four water and sediment samples were collected during summer 2009 (Figure 1) from Lakes Mariout (n = 10), Edku ( $=9)$, El-Burullus ( $=12)$, El-Manzallah $(n=11)$, and El-Bardaweel $(n=12)$. Water samples were collected in duplicate $10 \mathrm{~cm}$ below water surface at each station, using a purified PVC Niskin's bottle (5 L). Water temperature, salinity and $\mathrm{pH}$ were measured during the time of sampling using Hydrolab model (Mutlti Set 430i WTW) after previous calibration.

Transparency was measured using $25 \mathrm{~cm}$ diameter enameled Secchi disk. Total suspended matter was 


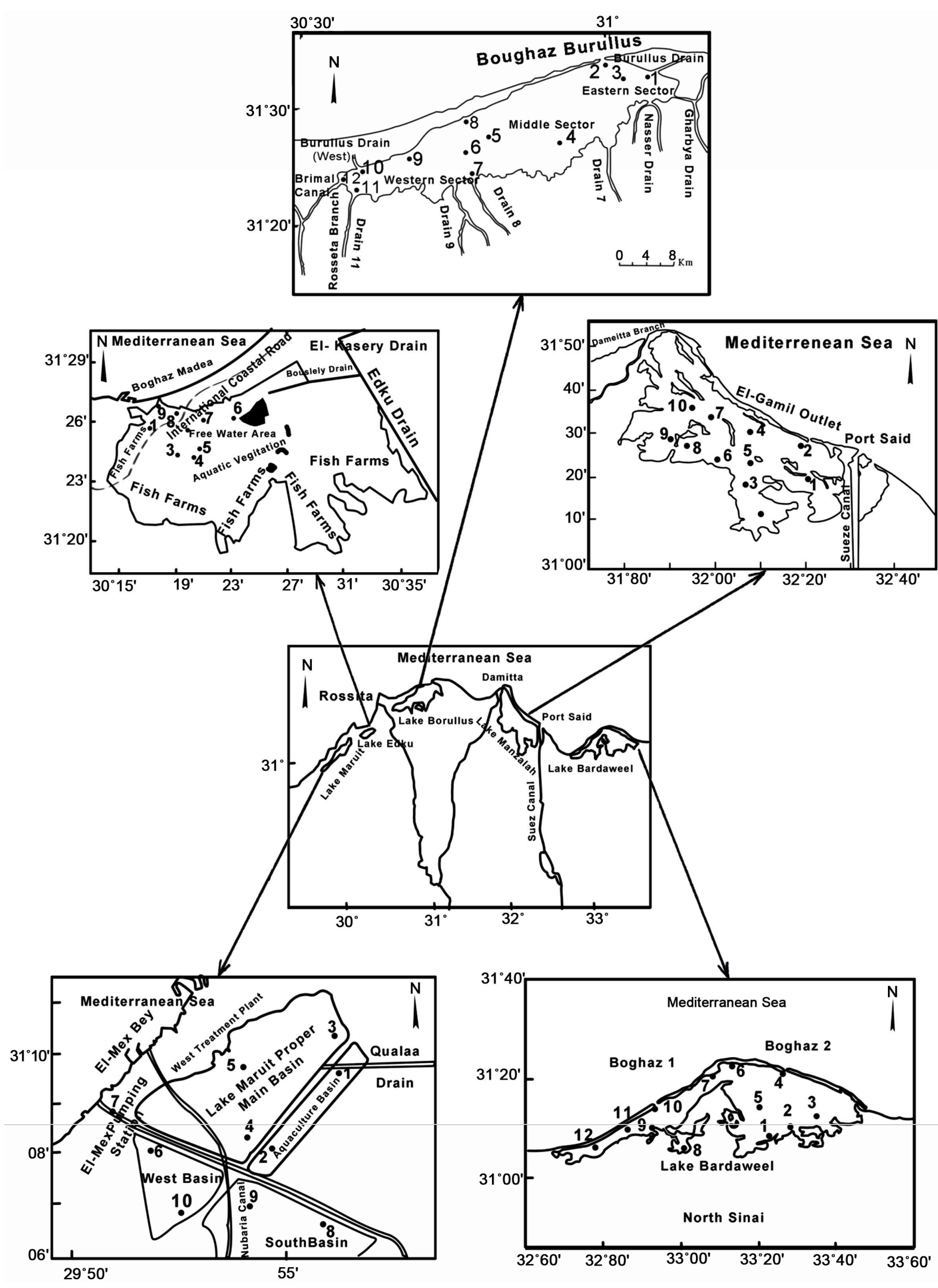

Figure 1. Map showing the positions of sampling stations of the five Egyptian northern lakes. 
obtained from one liter water sample then filtered through washed, dried and weighed $0.45 \mu \mathrm{m}$ membrane filters. The filters with its contents were kept for drying in the oven at $105^{\circ} \mathrm{C}$ until constant weight. The difference in the dry weight of filters before and after filtration was expressed in $\mathrm{mg} / \mathrm{l}$ suspended matter [22]. Dissolved oxygen (DO) was determined at the same day of collection using the classical Winkler method [23]. Biological Oxygen Demand (BOD) was measured using 5-day method as DO and oxidizable organic matter (OOM) was measured according to FAO [24]. Sediment samples were collected using a Van-Veen grab sampler. Locations were selected taking into consideration the expected affected areas by industrial and human activities. Samples were divided into two sub-samples, one for determination of OTC, placed in polypropylene bottles and the second one for determination of total tin, placed in pre-cleaned plastic bags. Sediment samples were kept frozen at $-20^{\circ} \mathrm{C}$ until analysis. Once in the laboratory the sediment samples were freeze-dried by using Labconco (England) freeze-drier, ground with agate mortar and stored at room temperature. For total tin analysis, an exact weight $\left(0.5 \mathrm{~g}\right.$ ) of a dry sediment sample was completely digested in Teflon vessels using a mixture of $\mathrm{HNO}_{3}$, $\mathrm{HF}$ and $\mathrm{HClO}_{4}$ (3:2:1) at $70^{\circ} \mathrm{C}$ [25]. The final solution was diluted to $25 \mathrm{ml}$ with double deionized distilled water. All digested solutions were analyzed in duplicate using an atomic absorption spectrometer (AAS Shimadzu Model AA 6800 equipped with a Graphite furnace automizer GFA-EX7) and the results were expressed in $\mu \mathrm{g} / \mathrm{g}$ dry wt. Conditions for GFA-EX7 obtained for tin applying AAS instrument were as follows: The absorption wavelength was $286.3 \mathrm{~nm}$, Slit width was 0.5 , ash temp was $400^{\circ} \mathrm{C}$, Atomizing temp was $2500^{\circ} \mathrm{C}$, Graphite tube was pyrolytic and detection limit was $2 \mathrm{ng} / \mathrm{g}$. All reagents used were of analytical grade (Merck). For quality control (QC) and quality assurance (QA), replicates were analyzed under same procedures mentioned above. A standard reference material (IAEA-356, marine sediment; Analytical Quality Control Services, Austria) was also digested and analyzed similarly to ensure the quality control and accuracy of the analysis (Table 1). Sn pollution levels in the collected sediments were measured using the Index of Geo-accumulation (Igeo) which consists of six grades (Tables 2(a)-(e)). The highest grade reflects 100 -fold metal concentration relative to background values: Igeo $=\log _{2}[\mathrm{Cn} /(1.5 \times \mathrm{Bn})]$ Where $\mathrm{Cn}$ is the measured concentration of element $\mathrm{n}$ in sediment sample, and $\mathrm{Bn}$ is the concentration of element $\mathrm{n}$. A factor of 1.5 is used because of possible variations in background data due to lithogenic effects [26]. OTC were determined as follows according to Tsuda et al., [27].

\section{Results and Discussion}

\subsection{Physicochemical Characteristics}

Depths, water temperature, transparency, electrical conductivity, salinity, $\mathrm{pH}, \mathrm{DO}, \mathrm{BOD}$, and OOM for each lake are listed in Tables 2(a)-(e). Depths varied between $0.25 \mathrm{~m}$ measured at station 4 of lake El-Burullus and $4.5 \mathrm{~m}$ measured at station 9 of lake Edku, revealing the shallowness of the area. Water temperature varied in a narrow range depending on the mean of daily number of sunshine hours and the time of sampling. The minimum value of $27.0^{\circ} \mathrm{C}$ was observed at station 1 of Lake El-Bardaweel, while the maximum value $31.2^{\circ} \mathrm{C}$ was measured at station 9 of Lake El-Manzalah. The water transparency is generally low due to the shallowness of these lakes and the continuous disturbance of the mud bottom by wind actions [28]. Transparency reached maximum value $(200 \mathrm{~cm})$ at stations $10 \& 11$ of Lake El-Bardaweel, while the minimum value $(12 \mathrm{~cm})$ was measured at station 1 of Lake El-Bardaweel revealing its turbid water. Transparency reached maximum value $(200 \mathrm{~cm})$ at Lake El-Bardaweel, while the minimum value $(12 \mathrm{~cm})$ was measured at Lake El-Bardaweel revealing its turbid water. The discharge of drainage water from different drains into the lakes, the invasion of sea water through the lake sea connection, the rates of evaporation and the rain water during winter are the main factors that affect the salinity of different lake water [29]-[32]. Water salinity varied in a very wide range. The maximum value of 67.7\%o was measured at station 12 of Lake El-Bardaweel. Worth mentioning that the hypersaline conditions which

Table 1. Results of validation study for Tin (Sn) concentration in reference material (IAEA356) analyzed together with sediments of the study area.

\begin{tabular}{ccccccc}
\hline Element & LOD & Certified value & Found & SD & Recovery\% & 95\% confidence interval \\
\hline Sn & $0.3 \mathrm{ppb}$ & $52.5 \mathrm{ppm}$ & $50.1 \mathrm{ppm}$ & 3.700 & 95.4 & $46.9-54.3$ \\
\hline
\end{tabular}

Mean concentration of certified value associated with the mean concentration (found) at 95\% confidence interval of $\mathrm{Sn}$ in sediments $(n=6)$. SD: Standarddeviation; LOD: Limit of detection. 
Table 2. (a) Physicochemical characteristics and concentrations of T-Sn, DBT, TBT and TPhT in lake Mariout during summer 2009; (b) Physicochemical characteristics and concentrations of T-Sn, DBT, TBT and TPhT in lake Edku during summer 2009; (c) Physicochemical characteristics and concentrations of T-Sn, DBT, TBT and TPhT in Lake El-Brullus during summer 2009; (d) Physicochemical characteristics and concentrations of T-Sn, DBT, TBT and TPhT in Lake El-Manzallah during summer 2009; (e) Physicochemical characteristics and concentrations of T-Sn, DBT, TBT and TPhT in lake El Bardaweel during summer 2009.

(a)

\begin{tabular}{|c|c|c|c|c|c|c|c|c|c|c|c|c|c|c|c|c|c|}
\hline \multicolumn{9}{|c|}{ Physicochemical characteristics of water } & \multicolumn{9}{|c|}{ Organotin compounds in sediments } \\
\hline Stations & $\begin{array}{l}\text { Depth } \\
\text { (m) } \\
\mathrm{cm}\end{array}$ & $\begin{array}{l}\text { Trans } \\
(\mathrm{cm}) \\
\mathrm{cm}\end{array}$ & $\begin{array}{c}\text { Temp } \\
\left({ }^{\circ} \mathrm{C}\right) \\
{ }^{\circ} \mathrm{C}\end{array}$ & $\begin{array}{c}\mathrm{EC} \mu \mathrm{S} / \mathrm{cm} \\
\mu \mathrm{S} / \mathrm{cm}\end{array}$ & Sal\%o & $\mathrm{pH}$ & $\begin{array}{c}\mathrm{DO} \\
\mathrm{mg} / \mathrm{l}\end{array}$ & $\begin{array}{l}\text { BOD } \\
\mathrm{mg} / \mathrm{l}\end{array}$ & $\begin{array}{c}\mathrm{OOM} \\
\mathrm{mgO}_{2} / \mathrm{l}\end{array}$ & $\begin{array}{l}\text { T-Sn } \\
\mu \mathrm{g} / \mathrm{g}\end{array}$ & $\begin{array}{l}\text { DBT } \\
\mu \mathrm{g} / \mathrm{g}\end{array}$ & $\begin{array}{l}\text { TBT } \\
\mu \mathrm{g} / \mathrm{g}\end{array}$ & $\begin{array}{l}\text { TPhT } \\
\mu \mathrm{g} / \mathrm{g}\end{array}$ & $\Sigma \mathrm{OT}$ & TBT/DBT & $\underset{\mathrm{T}-\mathrm{On} \%}{\sum \mathrm{OT} /}$ & Igeo \\
\hline 1 & 1.0 & 25 & 29.3 & 11.32 & 6.5 & 8.28 & 4.0 & 15.6 & 23.6 & 19.98 & $\mathrm{Nd}$ & 1.04 & $\mathrm{Nd}$ & 1.04 & - & 5 & 2.5 \\
\hline 2 & 1.5 & 25 & 28.9 & 12.92 & 7.5 & 8.38 & 5.6 & 16.9 & 28.8 & 21.96 & 0.44 & 0.84 & 0.34 & 1.62 & 1.91 & 7 & 2.7 \\
\hline 3 & 2.0 & 35 & 29.6 & 2.38 & 1.1 & 7.26 & 0.0 & 41.5 & 63.6 & 19.71 & $\mathrm{Nd}$ & 0.14 & $\mathrm{Nd}$ & 0.14 & - & 1 & 2.5 \\
\hline 4 & 1.8 & 42 & 27.8 & 5.18 & 2.8 & 7.86 & 2.4 & 31.2 & 55.4 & 15.57 & 0.21 & 0.88 & $\mathrm{Nd}$ & 1.09 & 4.19 & 7 & 2.2 \\
\hline 5 & 2.1 & 15 & 28.0 & 3.29 & 1.6 & 7.86 & 2.6 & 16.5 & 42.2 & 16.65 & $\mathrm{Nd}$ & 2.14 & $\mathrm{Nd}$ & 2.14 & - & 13 & 2.3 \\
\hline 6 & 3.4 & 35 & 28.1 & 8.69 & 4.9 & 8.64 & 10.0 & 16.5 & 22.1 & 26.55 & 1.74 & 1.72 & 0.22 & 3.68 & 0.99 & 14 & 2.9 \\
\hline 7 & 1.5 & 60 & 27.8 & 5.65 & 3.1 & 7.66 & 2.6 & 15.7 & 24.3 & 22.05 & $\mathrm{Nd}$ & 0.87 & $\mathrm{Nd}$ & 0.87 & - & 4 & 2.7 \\
\hline 8 & 0.7 & 40 & 29.1 & 4.68 & 2.5 & 7.92 & 3.5 & 12.6 & 26.4 & 19.26 & 0.05 & 0.29 & $\mathrm{Nd}$ & 0.34 & 5.80 & 2 & 2.5 \\
\hline 9 & 1.5 & 100 & 27.8 & 8.02 & 4.5 & 7.95 & 6.4 & 18.6 & 28.3 & 15.48 & 2.11 & 2.29 & 0.63 & 5.03 & 1.09 & 32 & 2.2 \\
\hline 10 & 0.8 & 20 & 30.2 & 8.70 & 4.9 & 8.37 & 13.0 & 16.8 & 28.9 & 22.51 & 1.78 & 1.25 & 0.39 & 3.42 & 0.70 & 15 & 2.7 \\
\hline Max. & 3.4 & 100 & 30.2 & 12.9 & 7.5 & 8.64 & 13.0 & 41.5 & 63.6 & 26.55 & 2.11 & 2.29 & 0.63 & 5.03 & 5.80 & 32 & 2.9 \\
\hline Min. & 0.7 & 15 & 27.8 & 2.38 & 1.1 & 7.26 & 0.0 & 12.6 & 22.1 & 15.48 & $\mathrm{Nd}$ & 0.14 & $\mathrm{Nd}$ & 0.14 & 0.70 & 1 & 2.2 \\
\hline Average & 1.6 & 40.0 & 28.7 & 7.8 & 3.9 & 8.02 & 5.01 & 20.2 & 34.4 & 19.97 & 1.06 & 1.15 & 0.40 & 2.61 & 1.08 & 13 & 2.5 \\
\hline SD & 0.8 & 24.8 & 0.9 & 3.4 & 2.4 & 0.4 & 3.9 & 9.0 & 14.5 & 3.5 & 0.92 & 0.7 & 0.2 & 1.6 & 2.1 & 9.2 & 0.2 \\
\hline
\end{tabular}

(b)

\begin{tabular}{|c|c|c|c|c|c|c|c|c|c|c|c|c|c|c|c|c|c|}
\hline Stations & $\begin{array}{l}\text { Depth } \\
\text { (m) } \\
\text { cm }\end{array}$ & $\begin{array}{l}\text { Tras } \\
(\mathrm{cm}) \\
\mathrm{cm}\end{array}$ & $\begin{array}{c}\text { Temp } \\
\left({ }^{\circ} \mathrm{C}\right) \\
{ }^{\circ} \mathrm{C}\end{array}$ & $\begin{array}{c}\text { EC } \\
\mu \mathrm{S} / \mathrm{cm}\end{array}$ & Sal\%o & $\mathrm{pH}$ & DO mg/l & $\begin{array}{c}\text { BOD } \\
\mathrm{mg} / \mathrm{l}\end{array}$ & $\begin{array}{c}\mathrm{OOM} \\
\mathrm{mgO}_{2} / \mathrm{l} \\
\mathrm{mg} / \mathrm{l}\end{array}$ & $\begin{array}{l}\text { T-Sn } \\
\mu \mathrm{g} / \mathrm{g}\end{array}$ & $\begin{array}{l}\text { DBT } \\
\mu \mathrm{g} / \mathrm{g}\end{array}$ & $\begin{array}{l}\text { TBT } \\
\mu \mathrm{g} / \mathrm{g}\end{array}$ & $\begin{array}{l}\text { TPhT } \\
\mu \mathrm{g} / \mathrm{g}\end{array}$ & $\Sigma \mathrm{OT}$ & TBT/DBT & $\begin{array}{c}\sum \mathrm{OT} / \\
\mathrm{T}-\mathrm{Sn} \%\end{array}$ & Igeo \\
\hline 1 & 1.5 & 30 & 27.2 & 3.58 & 1.8 & 8.57 & 8.0 & 6.2 & 13.9 & 16.7 & 2.04 & 2.63 & 0.81 & 5.48 & 1.29 & 33 & 2.3 \\
\hline 2 & 0.9 & 30 & 27.7 & 2.41 & 1.1 & 8.56 & 7.6 & 7.2 & 10.2 & 22.68 & 1.31 & 2.41 & $\mathrm{Nd}$ & 3.72 & 1.84 & 16 & 2.7 \\
\hline 3 & 1.0 & 32 & 28.7 & 2.33 & 1.1 & 8.75 & 8.2 & 7.6 & 10.3 & 24.41 & 1.3 & 4.88 & Nd & 6.18 & 3.75 & 25 & 2.8 \\
\hline 4 & 2.0 & 40 & 28.1 & 2.52 & 1.2 & 8.71 & 8.5 & 6.4 & 9.6 & 18.36 & 2.02 & 4.03 & $\mathrm{Nd}$ & 6.05 & 2.00 & 33 & 2.4 \\
\hline 5 & 2.1 & 50 & 28.1 & 2.28 & 1.0 & 8.32 & 8.5 & 6.2 & 10.6 & 17.46 & 0.91 & 1.34 & 0.68 & 2.93 & 1.47 & 17 & 2.3 \\
\hline 6 & 1.8 & 45 & 27.9 & 1.53 & 0.6 & 7.68 & 2.7 & 8.5 & 16.8 & 12.06 & 0.66 & 3.4 & $\mathrm{Nd}$ & 4.06 & 5.15 & 34 & 1.8 \\
\hline 7 & 1.8 & 45 & 28.5 & 1.61 & 0.6 & 8.32 & 9.3 & 6.4 & 14.6 & 23.94 & 2.81 & 3.98 & $\mathrm{Nd}$ & 6.79 & 1.42 & 28 & 2.8 \\
\hline 8 & 0.7 & 35 & 28.0 & 2.03 & 0.9 & 8.44 & 7.0 & 6.4 & 10.6 & 17.28 & 0.64 & 1.27 & 0.77 & 2.68 & 1.98 & 16 & 2.3 \\
\hline 9 & 4.5 & 30 & 29.4 & 2.01 & 0.9 & 8.87 & 18.2 & 17.2 & 22.1 & 18.99 & 0.67 & 1.65 & 0.74 & 3.06 & 2.46 & 16 & 2.5 \\
\hline Max. & 0.7 & 50 & 29.4 & 3.58 & 1.8 & 8.87 & 18.2 & 17.2 & 22.1 & 24.41 & 2.81 & 4.88 & 0.81 & 6.79 & 5.15 & 34 & 2.8 \\
\hline Min. & 4.5 & 30 & 27.2 & 1.53 & 0.6 & 7.68 & 2.7 & 6.2 & 9.6 & 12.06 & 0.64 & 1.27 & $\mathrm{Nd}$ & 2.93 & 1.29 & 16 & 1.8 \\
\hline Average & 2.0 & 38.0 & 28.2 & 2.26 & 1.0 & 8.47 & 8.67 & 8.0 & 13.2 & 19.09 & 1.37 & 2.84 & 0.33 & 4.54 & 2.07 & 24 & 2.4 \\
\hline SD & 1.1 & 7.8 & 0.6 & 0.60 & 0.4 & 0.4 & 4.0 & 3.5 & 4.2 & 4.0 & 0.8 & 1.3 & 0.1 & 1.6 & 1.3 & 8.1 & 0.3 \\
\hline
\end{tabular}


(c)

\begin{tabular}{|c|c|c|c|c|c|c|c|c|c|c|c|c|c|c|c|c|c|}
\hline Stations & $\begin{array}{l}\text { Depth } \\
(\mathrm{m}) \\
\mathrm{cm}\end{array}$ & $\begin{array}{l}\text { Trans } \\
(\mathrm{cm}) \\
\mathrm{cm}\end{array}$ & $\begin{array}{l}\text { Temp } \\
\left({ }^{\circ} \mathrm{C}\right) \\
{ }^{\circ} \mathrm{C}\end{array}$ & $\begin{array}{c}\text { EC } \\
\mu S / c m \\
\mu S / c m\end{array}$ & Sal\%o & $\mathrm{pH}$ & $\begin{array}{c}\mathrm{DO} \\
\mathrm{mg} / \mathrm{l}\end{array}$ & $\begin{array}{l}\text { BOD } \\
\mathrm{mg} / \mathrm{l}\end{array}$ & $\begin{array}{c}\mathrm{OOM} \\
\mathrm{mgO}_{2} / \mathrm{l} \\
\mathrm{mg} / \mathrm{l}\end{array}$ & $\begin{array}{l}\text { T-Sn } \\
\mu \mathrm{g} / \mathrm{g}\end{array}$ & $\begin{array}{l}\text { DBT } \\
\mu \mathrm{g} / \mathrm{g}\end{array}$ & $\begin{array}{l}\text { TBT } \\
\mu \mathrm{g} / \mathrm{g}\end{array}$ & $\begin{array}{l}\text { TPhT } \\
\mu \mathrm{g} / \mathrm{g}\end{array}$ & $\Sigma \mathrm{OT}$ & $\begin{array}{l}\text { TBT/ } \\
\text { DBT }\end{array}$ & $\sum \mathrm{OT} / \mathrm{T}-\mathrm{Sn} \%$ & Igeo \\
\hline 1 & 0.50 & 30 & 29.7 & 4.68 & 2.5 & 8.26 & 7.2 & 5.6 & 25.5 & 22.05 & 1.46 & 1.26 & $\mathrm{Nd}$ & 2.72 & 0.86 & 12 & 2.7 \\
\hline 2 & 3.0 & 40 & 30.5 & 8.36 & 4.7 & 8.35 & 7.6 & 5.4 & 23.4 & 14.4 & 2.21 & 0.21 & 2.07 & 4.49 & 0.10 & 31 & 2.1 \\
\hline 3 & 1.3 & 35 & 29.3 & 5.28 & 2.8 & 8.94 & 8.9 & 7.6 & 20.6 & 21.96 & 2.29 & 0.16 & 2.11 & 4.56 & 0.07 & 21 & 2.7 \\
\hline 4 & 2.5 & 20 & 30.3 & 4.60 & 2.4 & 8.77 & 6.6 & 5.0 & 23.5 & 31.59 & 1.64 & 0.48 & 1.29 & 3.41 & 0.29 & 11 & 3.2 \\
\hline 5 & 1.5 & 30 & 28.9 & 3.80 & 1.9 & 8.75 & 7.2 & 6.0 & 20.6 & 22.32 & 0.35 & 0.73 & 1.51 & 2.59 & 2.09 & 12 & 2.7 \\
\hline 6 & 1.2 & 18 & 29.0 & 3.30 & 1.6 & 8.44 & 6.6 & 5.6 & 17.6 & 32.22 & 1.49 & 1 & 1.23 & 3.72 & 0.67 & 12 & 3.2 \\
\hline 7 & 1.2 & 30 & 29.9 & 3.57 & 1.8 & 8.30 & 7.6 & 5.6 & 21.8 & 22.5 & 2.85 & 1.35 & 3.33 & 7.53 & 0.47 & 33 & 2.7 \\
\hline 8 & 1.0 & 30 & 29.0 & 2.78 & 1.3 & 8.74 & 7.2 & 6.5 & 22.5 & 25.83 & 2.34 & 0.32 & 3.83 & 6.49 & 0.14 & 25 & 2.9 \\
\hline 9 & 1.2 & 35 & 29.3 & 2.02 & 0.9 & 8.70 & 7.2 & 6.5 & 22.5 & 23.49 & 3.32 & 0.39 & 3.96 & 7.67 & 0.12 & 33 & 2.8 \\
\hline 10 & 1.3 & 50 & 29.1 & 1.25 & 0.4 & 8.65 & 7.4 & 4.8 & 22.8 & 21.87 & 2.15 & 0.76 & 1.07 & 3.98 & 0.35 & 18 & 2.7 \\
\hline 11 & 1.4 & 60 & 29.3 & 1.07 & 0.3 & 8.54 & 7.5 & 4.5 & 19.6 & 20.16 & 0.11 & 0.23 & 1.62 & 1.96 & 2.09 & 10 & 2.5 \\
\hline 12 & 1.10 & 70 & 30.0 & 1.15 & 0.4 & 8.92 & 8.2 & 6.1 & 17.5 & 25.38 & 0.78 & 1.34 & 0.93 & 3.05 & 1.72 & 12 & 2.9 \\
\hline Max. & 3.0 & 70 & 30.5 & 8.36 & 4.7 & 8.94 & 8.9 & 7.6 & 23.5 & 32.22 & 3.32 & 1.35 & 3.96 & 7.67 & 2.09 & 33 & 2.9 \\
\hline Min. & 0.25 & 18 & 28.9 & 1.07 & 0.3 & 8.26 & 6.6 & 4.5 & 17.5 & 14.40 & 0.11 & 0.16 & $\mathrm{Nd}$ & 1.96 & 0.07 & 11 & 2.1 \\
\hline Average & 1.3 & 37.3 & 29.5 & 3.49 & 1.8 & 8.61 & 7.43 & 5.77 & 21.49 & 23.65 & 1.75 & 0.69 & 2.09 & 4.53 & 0.39 & 19 & 2.76 \\
\hline SD & 0.7 & $15 . .52$ & 0.5 & 2.1 & 1.3 & 0.2 & 0.6 & 0.85 & 2.4 & 4.8 & 1.0 & 0.5 & 1.1 & 1.9 & 0.8 & 9.13 & 0.3 \\
\hline
\end{tabular}

(d)

\begin{tabular}{|c|c|c|c|c|c|c|c|c|c|c|c|c|c|c|c|c|c|}
\hline Stations & $\begin{array}{c}\text { Depth } \\
\text { (m) } \\
\mathrm{cm}\end{array}$ & $\begin{array}{c}\text { Trans } \\
(\mathrm{cm}) \\
\mathrm{cm}\end{array}$ & $\begin{array}{c}\text { Temp } \\
\left({ }^{\circ} \mathrm{C}\right) \\
{ }^{\circ} \mathrm{C}\end{array}$ & $\begin{array}{c}\mathrm{EC} \mu \mathrm{S} / \mathrm{cm} \\
\mu \mathrm{S} / \mathrm{cm}\end{array}$ & Sal\%o & $\mathrm{pH}$ & DO mg/l & BOD mg/l & $\begin{array}{c}\mathrm{OOM} \\
\mathrm{mgO}_{2} / \mathrm{l} \\
\mathrm{mg} / \mathrm{l}\end{array}$ & $\begin{array}{l}\text { T-Sn } \\
\mu \mathrm{g} / \mathrm{g}\end{array}$ & $\begin{array}{l}\text { DBT } \\
\mu \mathrm{g} / \mathrm{g}\end{array}$ & $\begin{array}{l}\text { TBT } \\
\mu \mathrm{g} / \mathrm{g}\end{array}$ & $\begin{array}{l}\text { TPhT } \\
\mu g / g\end{array}$ & $\sum \mathrm{OT}$ & TBT/DBT & $\begin{array}{c}\sum \mathrm{OT} / \\
\mathrm{T}-\mathrm{Sn} \%\end{array}$ & Igeo \\
\hline 1 & 0.4 & 12 & 28.4 & 5.3 & 2.9 & 7.8 & ND & 24.6 & 34.3 & 18.72 & 1.67 & 1.47 & 1.46 & 4.6 & 0.88 & 25 & 2.4 \\
\hline 2 & 1.0 & 33 & 28.7 & 13.5 & 7.9 & 8.5 & 6.4 & 3.2 & 14 & 19.15 & 1.36 & 1.25 & $\mathrm{Nd}$ & 2.61 & 0.92 & 14 & 2.5 \\
\hline 3 & 1.1 & 32 & 28.0 & 5.1 & 2.7 & 7.9 & 6.8 & 12.8 & 19.3 & 18.72 & 1.48 & 1.75 & 0.83 & 4.06 & 1.18 & 22 & 2.4 \\
\hline 4 & 0.9 & 50 & 30.2 & 17.5 & 10.4 & 8.6 & 7.2 & 6.4 & 22.9 & 24.21 & 1.5 & 3.27 & 1.24 & 6.01 & 2.18 & 25 & 2.8 \\
\hline 5 & 0.9 & 44 & 28.3 & 4.1 & 2.1 & 7.9 & 8.6 & 5.2 & 19.8 & 17.91 & 0.52 & 1.45 & $\mathrm{Nd}$ & 1.97 & 2.79 & 11 & 2.4 \\
\hline 6 & 1.3 & 75 & 30.6 & 3.5 & 1.8 & 8.6 & 7.2 & 4.6 & 21.3 & 19.08 & 0.6 & 0.98 & $\mathrm{Nd}$ & 1.58 & 1.63 & 8 & 2.5 \\
\hline 7 & 1.2 & 50 & 29.7 & 32.2 & 20.2 & 8.6 & 8.6 & 3.6 & 19.8 & 23.67 & 0.77 & 3.93 & $\mathrm{Nd}$ & 4.7 & 5.10 & 20 & 2.8 \\
\hline 8 & 1.2 & 70 & 31.1 & 6.9 & 3.8 & 8.7 & 7.4 & 4.2 & 16.4 & 26.64 & 1.31 & 4.61 & $\mathrm{Nd}$ & 5.92 & 3.52 & 22 & 2.9 \\
\hline 9 & 1.2 & 52 & 31.2 & 4.0 & 2.1 & 9.2 & 6.2 & 5.2 & 16.6 & 22.59 & 1.23 & 3.7 & 1.68 & 6.61 & 3.01 & 29 & 2.7 \\
\hline 10 & 1.5 & 50 & 31.1 & 17.0 & 10.2 & 8.3 & 8.2 & 4.1 & 17.2 & 19.89 & 0.66 & 3.37 & $\mathrm{Nd}$ & 4.03 & 5.11 & 20 & 2.5 \\
\hline 11 & 0.5 & 25 & 28.0 & 6.8 & 3.7 & 8.2 & 3.2 & 14.8 & 28.3 & 22.68 & 0.63 & 3.83 & $\mathrm{Nd}$ & 4.46 & 6.08 & 20 & 2.7 \\
\hline Max. & 0.4 & 75 & 31.2 & 32.2 & 10.4 & 9.2 & 8.6 & 24.6 & 34.3 & 26.64 & 1.67 & 4.61 & 1.68 & 7.96 & 6.08 & 25 & 2.9 \\
\hline Min. & 1.5 & 12 & 28.0 & 3.5 & 1.8 & 7.8 & 3.2 & 3.2 & 14 & 17.91 & 0.52 & 0.98 & Nd & 1.58 & 0.88 & 11 & 2.4 \\
\hline Average & 1.1 & 44.8 & 29.6 & 10.5 & 6.2 & 8.39 & 6.98 & 8.06 & 20.9 & 21.21 & 1.07 & 2.69 & 1.30 & 5.06 & 2.51 & 24 & 2.6 \\
\hline SD & 0.3 & 18.58 & 1.32 & 8.9 & 5.7 & 0.42 & 1.6 & 6.7 & 5.9 & 2.9 & 0.4 & 1.3 & 0.4 & 1.6 & 1.8 & 6.3 & 0.2 \\
\hline
\end{tabular}


(e)

\begin{tabular}{|c|c|c|c|c|c|c|c|c|c|c|c|c|c|c|c|c|c|}
\hline Stations & $\begin{array}{l}\text { Depth } \\
\text { (m) } \\
\mathrm{cm}\end{array}$ & $\begin{array}{l}\text { Trans } \\
(\mathrm{cm}) \\
\mathrm{cm}\end{array}$ & $\begin{array}{c}\text { Temp } \\
\left({ }^{\circ} \mathrm{C}\right) \\
{ }^{\circ} \mathrm{C}\end{array}$ & $\begin{array}{c}E C \\
\mu S / c m \\
\mu S / c m\end{array}$ & Sal\%o & $\mathrm{pH}$ & $\begin{array}{c}\mathrm{DO} \\
\mathrm{mg} / \mathrm{l}\end{array}$ & $\begin{array}{l}\text { BOD } \\
\text { mg/l }\end{array}$ & $\begin{array}{c}\mathrm{OOM} \\
\mathrm{mgO}_{2} / \mathrm{l} \\
\mathrm{mg} / \mathrm{l}\end{array}$ & $\begin{array}{l}\text { T-Sn } \\
\mu \mathrm{g} / \mathrm{g}\end{array}$ & $\begin{array}{l}\text { DBT } \\
\mu \mathrm{g} / \mathrm{g}\end{array}$ & $\begin{array}{l}\text { TBT } \\
\mu \mathrm{g} / \mathrm{g}\end{array}$ & $\begin{array}{l}\text { TPhT } \\
\mu \mathrm{g} / \mathrm{g}\end{array}$ & $\Sigma \mathrm{OT}$ & TBT/DBT & $\sum \mathrm{OT} / \mathrm{T}-\mathrm{Sn} \%$ & Igeo \\
\hline 1 & 1.3 & 125 & 27.0 & 81.40 & 57.7 & 8.42 & 8.8 & 3.6 & 4.7 & 16.62 & 0.28 & 3.22 & 0.89 & 4.39 & 11.50 & 26 & 2.3 \\
\hline 2 & 0.6 & 125 & 27.2 & 73.40 & 51.0 & 8.56 & 8.8 & 3.2 & 3.7 & 16.81 & 0.38 & 3.72 & 0.08 & 4.18 & 9.79 & 25 & 2.3 \\
\hline 3 & 0.9 & 70 & 28.1 & 81.20 & 57.4 & 8.66 & 9.1 & 3.8 & 4.8 & 15.47 & 0.69 & 2.99 & 0.62 & 4.3 & 4.33 & 28 & 2.2 \\
\hline 4 & 4.2 & 150 & 28.8 & 67.30 & 46.2 & 8.40 & 9.3 & 3.9 & 3.6 & 13.48 & 0.39 & 3.26 & 0.93 & 4.58 & 8.36 & 34 & 2.0 \\
\hline 5 & 1.9 & 60 & 28.6 & 76.00 & 53.2 & 8.50 & 8.5 & 3.3 & 3.2 & 11.97 & 0.47 & 2.97 & $\mathrm{Nd}$ & 3.44 & 6.32 & 29 & 1.8 \\
\hline 6 & 1.9 & 60 & 29.5 & 69.80 & 48.2 & 8.50 & 8.3 & 3.5 & 3.6 & 7.47 & 0.49 & 1.44 & 0.7 & 2.63 & 2.94 & 35 & 1.1 \\
\hline 7 & 0.9 & 60 & 29.3 & 70.40 & 48.7 & 8.46 & 9.1 & 3.2 & 3.3 & 7.38 & 0.48 & 0.55 & $\mathrm{Nd}$ & 1.03 & 1.15 & 14 & 1.1 \\
\hline 8 & 1.5 & 70 & 27.1 & 77.40 & 54.3 & 8.11 & 7.7 & 2.8 & 2.8 & 13.19 & 0.09 & 1.1 & $\mathrm{Nd}$ & 1.19 & 12.22 & 9 & 1.9 \\
\hline 9 & 0.5 & 120 & 27.7 & 65.00 & 44.5 & 8.24 & 8.5 & 2.9 & 3.0 & 10.06 & 0.64 & 1.12 & 0.48 & 2.24 & 1.75 & 22 & 1.5 \\
\hline 10 & 3.2 & 200 & 27.1 & 58.70 & 39.5 & 8.33 & 8.5 & 3.1 & 2.9 & 9.99 & 0.56 & 1.21 & 0.16 & 1.93 & 2.16 & 19 & 1.5 \\
\hline 11 & 1.9 & 200 & 27.2 & 74.20 & 53.2 & 8.33 & 7.5 & 3.3 & 4.0 & 10.16 & 0.71 & 1.11 & 0.61 & 2.43 & 1.56 & 24 & 1.6 \\
\hline 12 & 1.4 & 100 & 28.3 & 93.10 & 67.7 & 8.62 & 8.3 & 3.5 & 4.2 & 10.34 & 0.55 & 0.68 & 0.63 & 1.86 & 1.24 & 18 & 1.6 \\
\hline Max. & 4.2 & 200 & 29.5 & 39.10 & 67.7 & 8.66 & 9.3 & 3.1 & 4.7 & 16.81 & 0.71 & 3.72 & 0.93 & 5.36 & 12.22 & 35 & 2.3 \\
\hline Min. & 0.5 & 60 & 27.0 & 58.70 & 39.5 & 8.11 & 7.5 & 2.8 & 2.8 & 7.38 & 0.09 & 0.55 & $\mathrm{Nd}$ & 1.19 & 1.15 & 9 & 1.1 \\
\hline Average & 1.8 & 111.7 & 28.0 & 74.0 & 51.8 & 8.43 & 8.5 & 3.7 & 3.7 & 11.91 & 0.48 & 1.95 & 0.57 & 3.00 & 4.06 & 25 & 1.74 \\
\hline SD & 108.4 & 51.5 & 0.9 & 8.9 & 7.3 & 0.2 & 0.5 & 0.3 & 0.7 & 3.3 & 0.2 & 1.2 & 0.3 & 1.3 & 4.4 & 7.7 & 0.4 \\
\hline
\end{tabular}

prevailed in the area of station 12 is a result of the isolation of this area in addition to the high rate of evaporation. The minimum value $(0.3 \%$ ) was measured at station 11 . The water salinity at different areas revealed that the salinity decreased in the order: Bardaweel $>$ El-Manzalah $>$ Mariout $>$ El-Brullus $>$ Edku depending on the amount of drainage waters. Conductivity showed a similar trend as salinity during the study period. The maximum EC value $(93.10 \mathrm{mS} / \mathrm{cm})$ was observed at station 12 of Lake El-Bardaweel, while the lowest one (1.07 $\mathrm{mS} / \mathrm{cm}$ ) was measured at station 11 of Lake El-Brullus. The water of the Nile delta Lakes lies in alkaline side (Tables 2(a)-(e)). pH values ranged between 7.26 - 8.92. Station 3 of Lake Maruit sustained the lowest $\mathrm{pH}$ value, while station 3 of Lake El-Brullus maintained the maximum value. The average values of $\mathrm{pH}$ at different areas decreased in the order: El-Brullus $>$ Edku $>$ El-Bardaweel $>$ El-Manzalah $>$ Mariout. The obtained results of dissolved oxygen showed a complete depletion at station 3 of Lake Maruit, while the maximum of $18.2 \mathrm{mg} / \mathrm{l}$ was observed at station 9 of Lake Edku. The average values of DO at different areas decreased in the order: Edku > El-Bardaweel $>$ El-Brullus $>$ El-Manzalah $>$ Mariout. The obtained values of biological oxygen demand (BOD) were varied in a wide range. The minimum value $(2.8 \mathrm{mg} / \mathrm{l})$ was measured at station 8 of lake El-Bardaweel, while the maximum of $41.5 \mathrm{mg} / \mathrm{l}$ was measured at station 3 of lake Maruit. The average values of BOD at different areas revealed that the BOD decreased in the order: Mariout $>$ El-Manzalah $>$ Edku $>$ El-Brullus $>$ El-Bardaweel. The observed oxidizable organic matter varied also in a wide range between different Lakes (Tables 2(a)-(e)). The maximum value $\left(63.6 \mathrm{mgO}_{2} / \mathrm{l}\right)$ was measured at station 3 of Lake Mariout and the minimum $\left(2.8 \mathrm{mgO}_{2} / \mathrm{l}\right)$ was observed at station 8 of Lake El-Bardaweel. The average values of OOM at different areas decreased in the order: Mariout $>$ El-Burullus $>$ El-Manzalah $>$ Edku $>$ El-Bardaweel revealing high load of anthropogenic pollutants, i.e. agricultural, sewage and industrial in the lake Mariout [29].

\subsection{Total Tin Compounds}

The concentrations of total Sn (T-Sn) in sediments are shown in Tables 2(a)-(e). The results show that concentrations of T-Sn are scattered in the ranges of 15.48 - $26.55 \mu \mathrm{g} / \mathrm{g}$ dry wt, $12.06-24.41 \mu \mathrm{g} / \mathrm{g}$ dry wt, 14.4 - 32.22 
$\mu \mathrm{g} / \mathrm{g}$ dry wt, 17.91 - $26.64 \mu \mathrm{g} / \mathrm{g}$ dry wt and 7.38 - $16.81 \mu \mathrm{g} / \mathrm{g}$ dry wt for lakes Mariout, Edku, El-Brullus, ElManzallah and El-Bardaweel, respectively. The highest average value of T-Sn was measured in sediments of Lake El-Brullus due shipping activities, sewage and industrial wastes from different drains. A negative correlation ( $r=-0.67$ at $p>0.001$ ) was found between T-Sn and salinity (Table 4) is due to freshwater of the lakes, especially Edku, Mariout, El-Brullus, and El-Manzalah contaminated with sewage and industraial wastes from different drains. To determine whether the T-Sn concentrations pose a threat to aquatic life, T-Sn concentrations in sediments of the study area were compared to threshold limit value (TLV) of $30 \mu \mathrm{g} / \mathrm{g}$ dry wt. It is clear from Tables 2(a)-(e) that T-Sn concentrations surpassed the TLV only in lake El-Brullus at station 6 located in the western sector (Figure 1(d)). This station is affected mainly by fishing boats that utilizes OTC as antifouling paints as well as the discharge of drain (8) which is characterized by its high concentration of pesticides and heavy metals [27] [28]. Accumulation values (Igeo) for Sn in northern lakes (Tables 2(a)-(e)) cleared out that sediments of the five lakes are classified as very low to low polluted.

\subsection{Tributyl Tin Compounds}

Tributyl tin (TBT) concentrations in sediments (Tables 2(a)-(e)) varied widely depending on the location and ranged from 0.14 to $2.29 \mu \mathrm{g} / \mathrm{g}$ Sn dry wt, from 1.27 to $4.88 \mu \mathrm{g} / \mathrm{g}$ Sn dry wt, from 0.16 to $1.35 \mu \mathrm{g} / \mathrm{g}$ Sn dry wt, from 0.98 to $4.61 \mu \mathrm{g} / \mathrm{g}$ Sn dry wt; and from 0.55 to $3.72 \mu \mathrm{g} / \mathrm{g}$ Sn dry wt for lakes Mariout; Edku; El-Brullus; El-Manzallah and El-Bardaweel, respectively. It is clear that TBT is the predominant species of OTC in all lakes except lake El-Brullus (Tables 2(a)-(e)) with percentage to total OTC ranged from 32\% to 100\%. The present study cleared out that concentrations of TBT in investigated sediments are higher than that of its degradation where the ratio of TBT/DBT $>1$ in most samples. This indicates that TBT is still being introduced into the aquatic environment, most probably due to the use of antifouling paints.

The highest average values of TBT are measured in Lake Edku and El-Manzallah. The highest TBT concentrations measured in Lake Edku are probably due to the presence of thick layer of green phytoplankton and herbicides covering the surface of most investigated areas. The presence of such thick layer inhibits transmittance of UV and consequently decreasing photo-degradation process for TBT compounds compared to other lakes. Intensive fishing activities in the lake are leading to historical deposition of TBT compounds. Moreover, the spreading of large number of islands through the lake that helps in increasing human activities as a main anthropogenic source of TBT compounds. The three large drains El-Barseik, Edku and El-Boseily, in addition to a number of sub-drains connected with main drains are acting as additional sources of pollution with TBT compounds (Tables 2(a)-(e)). On the other hand, higher concentrations of TBT in sediments of lake El-Manzallah are resulted from large number of boats and intensive fishing activities, the water transparency is generally low at lake El-Manzallah $(0.45 \mathrm{~m})$ due to the continuous disturbance of the mud bottom by wind action (Tables 2(a)-(e)) revealing relatively low photo-degradation of TBT by sunlight. Impacts of different drains, i.e. Hadous, Ramsisand and Bahr El-Baqar that discharge large quantities of industrial, agricultural and domestic wastes are also considered.

The Australian sediment quality guidelines for TBT are $5 \mathrm{ng} / \mathrm{g}$ and $70 \mathrm{ng} / \mathrm{g}$ for low and high trigger values [33]. Sediments from different stations of the present study contain TBT concentrations higher than the highest trigger value. This confirms that these sediments may pose a threat to a benthic biota. High levels of TBT in sediment samples suggested its widespread contamination and could be indicative of the continuing usage of TBTbased antifouling paints on ship hulls. Dowson et al. [34] introduced a classification for TBT concentrations in sediments, characterizing concentrations below $3 \mathrm{ng} / \mathrm{g}$ as uncontaminated, 3 - $20 \mathrm{ng} / \mathrm{g}$ as light contaminated, 20 $100 \mathrm{ng} / \mathrm{g}$ as moderately contaminated, $100-500 \mathrm{ng} / \mathrm{g}$ as highly contaminated and above $500 \mathrm{ng} / \mathrm{g}$ as grossly contaminated. Comparing our results with this scheme, cleared out that sediments of the Egyptian northern lakes are highly to grossly contaminated by TBT. Relatively high concentrations of TBT in some sediments of different lakes may have a potential for future TBT release from the re-suspension of fine sediments. Page et al. [35] and Axiak et al. [36] showed soluble butyltins were released from heavily contaminated sediments. Comparing these concentrations to that observed in other areas around the world (Table 3) revealed that sediments of northern lakes contained TBT concentrations lower than that observed in other countries such as Sanricu Coast, Japan, North East Coast, Spain, Barcelona Harbor, Spain. However, Aegean Sea, Turkey, Mediterranean Sea, Egypt and Suez Gulf, Egypt showed lower TBT than northern lakes. 
Table 3. Comparison of DBT, TBT and TphT concentrations ( $\mu \mathrm{g} / \mathrm{g}$ Sn dry wt) in sediments of the present study to those reported in the literature for other regions of the world.

\begin{tabular}{|c|c|c|c|c|}
\hline Area & DBT & TBT & $\mathrm{TPhT}$ & References \\
\hline Egyptian Northern Lakes & $0.05-3.32$ & $0.16-4.61$ & & Present study \\
\hline Sanricu Coast, Japan & $1-3.4$ & $0.002-14$ & - & [38] \\
\hline North East Coast, Spain & $0.047-3.519$ & $0.051-7.673$ & - & {$[39]$} \\
\hline Barcelona Harbor, Spain & - & $0.098-4.702$ & - & {$[40]$} \\
\hline Aegean Sea, Turkey & - & Nd - 3.008 & - & [41] \\
\hline River Thames, UK & $0.012-0.219$ & - & - & {$[42]$} \\
\hline North West Coast, Spain & $0.0005-0.357$ & - & - & [43] \\
\hline Cadiz Coast, Spain & - & - & $0.015-0.940$ & {$[44]$} \\
\hline German North Sea and Baltic Sea Marinas & - & - & $0.015-3.540$ & [32] \\
\hline Scheldt Estuary, The Netherlands & - & - & $0.007-0.012$ & [45] \\
\hline Spanish Northeastern Coast & - & - & 12.27 & [46] \\
\hline Mediterranean Sea, Egypt & $\mathrm{Nd}-2.16$ & $\mathrm{Nd}-8.55$ & - & [10] \\
\hline Suez Gulf, Egypt & $0.07-2.27$ & $0.35-0.77$ & - & [47] \\
\hline
\end{tabular}

\subsection{Dibutyl Tin Compounds}

DBT concentrations in sediments of the northern lakes varied widely and ranged from 0.05 to $2.11 \mu \mathrm{g} / \mathrm{g}$ Sn dry wt, from 0.64 to $2.81 \mu \mathrm{g} / \mathrm{g}$ Sn dry wt, from 0.11 to $3.32 \mu \mathrm{g} / \mathrm{g}$ Sn dry wt, from 0.52 to $1.67 \mu \mathrm{g} / \mathrm{g}$ Sn dry wt and from 0.09 to $0.71 \mu \mathrm{g} / \mathrm{g}$ Sn dry wt for lakes Mariout, Edku, El-Brullus, El-Manzallah, and El-Bardaweel, respectively. Presence of DBT at most sampled sites of lakes indicates that there is an important photochemical and biological degradation process on these sediments, together with its mobilization. This idea is confirmed by a relative higher abundance of DBT in sediments of lake El-Brullus (Tables 2(a)-(e)). TBT/DBT ratios are found >1 confirms also an active degradation process. However, this ratio may also indicate that either TBT inputs to Lake El-Brullus are older or that new inputs are absent or negligible than TBT degradation rate [37]. In spite of the positive correlation ( $\mathrm{r}=0.45, p=0.001$ ) between DBT and T-Sn, there was no significant correlation between TBT and DBT $(r=-0.03, p=0.001)$ in sediments from the five northern lakes (Table 4). The absence of such correlation might give clear evidence that both compounds did not come from the same source. This finding confirms the slow rate of degradation of TBT in sediments. On the other hand, a negative correlation $(\mathrm{r}=$ $-0.48, p=0.001$ ) was found between DBT and water salinity (Table 4). The current research was not specifically designed to evaluate which factor strongly related to such variations in the distribution of these OTC.

Comparing concentrations of DBT to those reported in literature (Table 3) showed concentrations similar to others found in Sanricu coast, Japan and North east coast. In contrast, they were higher than the ranges reported for River Thames, UK, for North west coast Spain, for Mediterranean Sea, Egypt and for Suez Gulf, Egypt.

\subsection{Triphenyltin Compounds}

$\mathrm{TPhT}$ in sediments of the northern lakes were not observed in most sediments and its concentrations ranged from Nd to $0.63 \mu \mathrm{g} / \mathrm{g}$ Sn dry wt, Nd to $0.81 \mu \mathrm{g} / \mathrm{gSn}$ dry wt, Nd to $3.96 \mu \mathrm{g} / \mathrm{g}$ Sn dry wt, Nd to $1.68 \mu \mathrm{g} / \mathrm{g}$ Sn dry wt, and Nd-0.93 $\mu \mathrm{g} / \mathrm{g}$ Sn dry wt for lakes Mariout, Edku, El-Brullus, El-Manzallah and El-Bardaweel; respectively. Variations occurring among lakes depending on changes in chemical and physical characteristics of each lake, various sources of pollutants, degradation process and drains run-off. It is clear from Tables 2(a)-(e) that TphT is the predominant species in lake El-Brullus. It exhibited concentrations of TPhT range from Nd to 3.96 $\mu \mathrm{g} / \mathrm{g}$ Sn dry wt. This could be attributed to the presence of several numbers of drains, channels and sub-drains, i.e. drain west El-Burullus, El-Hoksa, Terra and Brimbal canal discharging a huge amounts of agriculture wastes containing TPhT which is used as fungicide in agriculture purposes into the lake. TPhT concentrations are decreasing in the following order: El-Burullus $>(2.59 \mu \mathrm{g} / \mathrm{g} \mathrm{Sn})>$ El-Manzallah $(1.30 \mu \mathrm{g} / \mathrm{g} \mathrm{Sn})>$ Edku $(0.75 \mu \mathrm{g} / \mathrm{g}$ $\mathrm{Sn})>$ El-Bardaweel $(0.57 \mu \mathrm{g} / \mathrm{g} \mathrm{Sn})>$ Mariout $(0.40 \mu \mathrm{g} \mu \mathrm{g} / \mathrm{g} \mathrm{Sn})$. Although a significant positive correlation $(\mathrm{r}=$ $0.67, p=0.001)$ was found between DBT and TPhT, a weak negative correlation $(\mathrm{r}=-0.37, p=0.001)$ was found between TBT and TPhT in sediments from northern lakes revealing different sources of pollution (Table 4). 
Table 4. Correlation cofficient (r) between physicochemical parameter of lake water and organotin compounds in sediments of all lakes.

\begin{tabular}{|c|c|c|c|c|c|c|c|c|c|c|c|c|}
\hline Parameters & Depth & Trans & Temp & EC & Salinity & $\mathrm{pH}$ & DO & OOM & BOD & DBT & TBT & TPhT \\
\hline T-Sn & -0.31 & -0.55 & 0.43 & -0.66 & -0.67 & 0.29 & -0.05 & 0.37 & 0.12 & 0.45 & 0.07 & 0.38 \\
\hline DBT & -0.15 & -0.37 & 0.22 & -0.48 & -0.48 & 0.19 & 0.09 & 0.26 & 0.06 & & -0.03 & 0.67 \\
\hline TBT & -0.05 & 0.10 & -0.05 & 0.10 & 0.09 & 0.17 & 0.13 & -0.29 & -0.21 & & & -0.37 \\
\hline TPhT & 0.15 & 0.38 & 0.44 & -0.41 & -0.41 & 0.22 & -0.24 & 0.37 & -0.12 & & & \\
\hline
\end{tabular}

Comparing concentrations of TphT to that reported in the Literature (Table 3) showed higher concentrations than those reported for Cadiz coast, Spain, for German North Sea and Baltic Sea marinas, and for Scheldt estuary, The Netherlands due to the extensive shipping activities and the discharge of sewage, agricultural and industrial effluents into Egyptian lakes. On contrast they were lower than those reported for Spanish northeastern coast.

The results in Tables 2(a)-(e) show that the highest average concentrations of total OTC were found in sediments of lake El-Manzallah (5.06), lake Edku (4.54), Lake El-Brullus (4.53) compared to Lake El-Bardaweel (3.0) and Lake Mariout (2.61) depending on the pollution sources. A percentage of total OTC to total tin concentration were ranged from $13 \%$ to $25 \%$ in sediments of the northern lakes. Lake Edku (24\%), lake El-Brullus (19\%), lake El-Manzallah (24\%) and Lake El-Bardaweel (25\%) were remarkably higher than Lake Mariout (13\%) as shown in Tables 2(a)-(e). Significant levels of OTC were found in sediments of these lakes.

Results of the present investigation show that the degree of pollution in the northern lakes is influenced by industrial and sewage discharges. Concentrations found of TBT, DBT and TPhT in the sediments are a consequence of the historical use of OTC, mainly related to fishing activities in the lakes. Regular monitoring of OTC pollution to assess hazardous effects for the aquatic life is recommended.

\section{Conclusion}

OTCs in the five northern lakes revealed some highly localized areas of contamination that were severe enough to cause harmful effects on marine flora and fauna. Levels of contamination are depending mainly on the shipping activity, changes in chemical and physical characteristics of each lake, various sources of pollutants, degradation process and drains run-off. No correlation between TBT and DBT in sediments demonstrated that both compounds did not come from the same source (i.e. antifouling paints), indicating their different deposition, and confirming the slow rate of degradation in sediments. The results concluded that sediments of the Egyptian northern lakes were highly to grossly contaminate by TBT. Among the five northern lakes, Lake El-Brullus shows the maximum concentration of $\sum$ OT. The high input of OTC particularly in sediments, overrides any removal by degradation pathway. Up to the present, no studies on biological effects have been done in this area. The present research concluded that TBT levels suggested that stringent legislative measures were needed to curb the usage of these contaminants in marine paints. Otherwise, OTC would have detrimental effect on our marine resources and ecosystem, which probably could not reverse.

\section{References}

[1] Blunden, S.J. and Chapman, A. (1984) In: Craig, P.J., Ed., Organometalic Compounds in the Environment. Principals and Reaction, Longman, Essex, 111.

[2] HSDB (2003) Environmental Standards and Regulations of Tin. National Library of Medicine, Hazardous Substances Data Bank, Bethesda.

[3] Alzieu, C., Michel, P., Tolosa, I., Bacci, E., Mee, L.D. and Readman, J.W. (1991) Organotin Compounds in the Medite. Rranean: A Containing Cause for Concern. Marine Environmental Research, 32, 261-270. http://dx.doi.org/10.1016/0141-1136(91)90047-C

[4] Shiryaev, V.I. and Storozhenko, P.A. (2012) Application of Organotin Compounds for Protecting Wood and Other Materials and in Nonfouling Paints. Polymer Science, Series D, Glues and Sealing Materials, 5, 221-230. http://dx.doi.org/10.1134/S1995421212030203 
[5] Almeida, A.C., Wagener, A.L.R., Maia, C.B. and Miekeley, N. (2004) Speciation of Organotin Compounds in Sediment Cores from Guanabara Bay, Rio de Janeiro (Brazil) by Gaschromatography-Pulsed Flame Photometric Detection. Applied Organometallic Chemistry, 18, 694-704. http://dx.doi.org/10.1002/aoc.661

[6] Maguire, R.J. (1996) The Occurrence, Fate and Toxicity of Tributyltin and Its Degradation Products in Fresh Water Environments. NWRI Contribution No. 94-106, National Water Research Institute, Canada.

[7] Alzieu, C. (1998) Tributyl Tin: Case Study of a Chronic Contaminant in the Coastal Environment. Ocean Coastal Management, 40, 23-36. http://dx.doi.org/10.1016/S0964-5691(98)00036-2

[8] Díez, S., Lacorte, S., Viana, P., Barceló, D. and Bayona, J.M. (2005) Survey of Organotin Compounds in Rivers and Coastal Environments in Portugal 1999-2000. Environmental Pollution, 136, 525-536. http://dx.doi.org/10.1016/j.envpol.2004.12.011

[9] Buggy, C.J. and Tobin, J.M. (2006) Seasonal and Spatial Distributions of Tributyltin in Surface Sediment of the Tolka Estuary. Dublin, Ireland. Environmental Pollution, 143, 294-303. http://dx.doi.org/10.1016/j.envpol.2005.11.025

[10] Hoch, M., Jacinto, A.A. and Martin, L. (2003) Assessment of Adsorption Behavior of Dibutyltin (DBT) to Clay-Rich Sediments in Comparison to the Highly Toxic Tributyltin (TBT). Environmental Pollution, 123, 217-227. http://dx.doi.org/10.1016/S0269-7491(02)00402-5

[11] HSE (1992) A Review of the Environment Effects of Triorganotin Compounds. Health and Safety Executive, Pesticide Registration Section, Advisory Committee on Pesticide, Report No. 111, Merseyside, 42.

[12] Biselli, S., Bester, K., Hühnerfuss, H. and Fent, K. (2000) Concentrations of the Antifouling Compound Irgarol 1051 and of Organotins in Water and Sediments of German North and Baltic Sea Marinas. Marine Pollution Bulletin, 40, 233-243. http://dx.doi.org/10.1016/S0025-326X(99)00177-0

[13] Fent, K. and Meier, W. (1994) Effects of Triphenyltin on Fish Early Life Stages. Archives of Environmental Contamination and Toxicology, 27, 224-231. http://dx.doi.org/10.1007/BF00214266

[14] Paton, G.I., Cheewasedtham, W., Marr, I.L. and Dawson, J.J.C. (2006) Degradation and Toxicity of Phenyltin Compounds in Soil. Environmental Pollution, 144, 746-751. http://dx.doi.org/10.1016/j.envpol.2006.02.024

[15] Said, T.O., Farag, R.S., Younis, A.M. and Shreadah, M.A. (2006) Organotin Species in Fish and Bivalves Samples Collected from the Egyptian Mediterranean Coast of Alexandria, Egypt. Bulletin of Environmental Contamination and Toxicology, 77, 451-458. http://dx.doi.org/10.1007/s00128-006-1086-8

[16] Shreadah, M.A., Said, T.O., Younis, A.M. and Farag, R.S. (2006) Speciation of Organotin Compounds in Sediments of Semi-Closed Areas along the Mediterranean Coast of Alexandria. Chemistry and Ecology, 22, 395-404. http://dx.doi.org/10.1080/02757540600917443

[17] Said, T.O., Shreadah, M.A., Abdelel Ghani, S.A. and Ahmed, A.M. (2010) Alkyltin and Alkayllead Compounds in Coastal Water of Suez Gulf, Egypt. Egyptian Journal of Aquatic Research, 36, 33-42.

[18] Hoch, M. (2001) Organotin Compounds in the Environment-An Overview. Applied Geochemistry, 16, 719-743. http://dx.doi.org/10.1016/S0883-2927(00)00067-6

[19] Said, T.O., Moselhy, K.M., Rashad, A.M. and Shreadah, M.A. (2008) Organochlorine Contaminants in Water, Sediment and Fish of Lake Burullus, Egyptian Mediterranean Sea. Bulletin of Environmental Contamination and Toxicology, 81, 136-146. http://dx.doi.org/10.1007/s00128-008-9422-9

[20] Shreadah, M.A., Said, T.O., Abdel Ghani, S.A. and Ahmed, A.M. (2008) Alkyllead and Alkyltin Species in Different Fishes Collected from the Suez Gulf, Egypt. Proceedings of the 2nd International Conference on Aquatic Research, Egyptian Journal of Aquatic Research, 34, 64-73.

[21] Abdel Ghani, S.A., Shobier, A.H., Said, T.O. and Shreadah, M.A. (2011) Organotin Compounds in Egyptian Mediterranean Sediments. Egyptian Journal of Aquatic Resources, 36, 221-229.

[22] Van der Linde, D. (1998) Protocol for Total Suspended Matter Estimate. JRC Technical Note.

[23] Strickland, J.D.H. and Parsons, T.R. (1975) A Practical Hand Book of Seawater Analysis. Bulletin Fisheries Research, Ottawa.

[24] FAO (1976) Mannual of Methods in Aquatic Environmental Research, Part I: Permanganate Value (Oxidiability) of Organic Matter in Natural Waters. FAO Fisheries Technical Paper, Vol. 137, 169-174.

[25] UNEP/IAEA (1986) References Methods for Marine Pollution Studies, Vol. 39. United Nations Environment Programme/The International Atomic Energy Agency.

[26] Müller, D. (1987) Comprehensive Trace Level Determination of Organotin Compounds in Environmental Samples Using High-Resolution Gas Chromatography with Flame Photometric Detection. Analytical Chemistry, 59, 617-623. http://dx.doi.org/10.1021/ac00131a017

[27] Tsuda, T., Nakanishi, H., Aoki, S. and Takebayashi, J. (1987) Determination of Butyltin and Phenyltin Compounds in 
Biological and Sediment Samples by Electron-Capture Gas Chromatography. Journal of Chromatography A, 387, 361370. http://dx.doi.org/10.1016/S0021-9673(01)94538-8

[28] Shriadah, M.A. (1991) Recent Observations on Some Hydrographic and Chemical Aspects of Lake Edku Waters, Egypt. Bulletin of the High Institute of Public Health, 22, 185-201.

[29] Shriadah, M.A. and Tayel, F.T. (1992) Impacts of Industrial, Sewage and Agricultural Effluents on Lake Edku and Abu-Qir Bay. Bulletin Faculty of Science, Alexandria University, Alexandria, 32, 103-155.

[30] Shriadah, M.A. and Okbah, M.A. (2003) Speciation of Iron, Manganese, Copper and Zinc in Sediments of Lake Burullus, Egypt. A. M. S. E. (France), 64, 49-61.

[31] Shreadah, M.A., Abdel Ghani, S.A., Abd El Samie, A., Ahmed, A.M. and Hawash, H.B.I. (2012) Mercury and Methyl Mercury in Sediments of Northern Lakes-Egypt. Journal of Environmental Protection, 3, 254-261. http://dx.doi.org/10.4236/jep.2012.33032

[32] Khalil, M.Kh., El Zokm, G.M., Fahmy, M.A., Said, T.O. and Shreadah, M.A. (2013) Geochemistry of Some Major and Trace Elements in Sediments of Edku and Mariut Lakes, North, Egypt. World Applied Sciences Journal, 24, $282-294$.

[33] ANZECC and ARMCANZ (2000) Australian and New Zealand Guidelines for Fresh and Marine Water Quality. Australian and New Zealand Environment Conservation Council and Agriculture and Resource Management Council of Australian and New Zealand, Canberra.

[34] Dowson, P.H., Bubb, J.M. and Lester, J.N. (1993) Temporal Distribution of Organotins in the Aquatic Environment: Five Years after the 1987 UK Retail Ban on TBT Based Antifouling Paints. Marine Pollution Bulletin, 26, 487-494. http://dx.doi.org/10.1016/0025-326X(93)90465-V

[35] Page, D.S., Ozbal, C.C. and Lanphear, M.E. (1996) Concentration of Butyltin Species in Sediments Associated with Shipyard Activity. Environmental Pollution, 91, 237-243. http://dx.doi.org/10.1016/0269-7491(95)00046-1

[36] Axiak, V., Vella, A.J., Agius, D., Bonnici, P., Cassar, G., Cassone, R., Chircop, P., Micallef, D., Mintoff, B. and Sammut, M. (2000) Evaluation of Environmental Levels and Biological Impact of TBT in Malta (Central Mediterranean). Science of the Total Environment, 258, 89-97. http://dx.doi.org/10.1016/S0048-9697(00)00509-X

[37] Ritsema, R., de Smaele, T., Moens, L., de Jong, A.S. and Donard, O.F.X. (1998) Determination of Butyltins in Harbor Sediment and Water by Aqueous Phase Ethylation GC-ICP-MS and Hydride Generation GC-AAS. Environmental Pollution, 99, 271-277. http://dx.doi.org/10.1016/S0269-7491(97)00128-0

[38] Harino, H., Yamamoto, Y., Eguchi, S., Kawai, S., Kurokawa, Y., Arai, T., Ohji, M., Okamura, H. and Miyazaki, N. (2007) Concentrations of Antifouling Biocides in Sediment and Mussel Samples Collected from Otsuchi Bay, Japan. Archives of Environmental Contamination and Toxicology, 52, 179-188. http://dx.doi.org/10.1007/s00244-006-0087-2

[39] Diez, S., Jover, E., Albaiges, J. and Bayona, J.M. (2006) Occurrence and Degradation of Butyltins and Waster Marker Compounds in Sediments from Barcelona Harbor, Spain. Environment International, 32, 858-865. http://dx.doi.org/10.1016/j.envint.2006.05.004

[40] Diez, S., Abalos, M. and Bayona, J.M. (2002) Organotin Contamination in Sediments from the Western Mediterranean Enclosures Following 10 Years of TBT Regulation. Water Research, 36, 905-918. http://dx.doi.org/10.1016/S0043-1354(01)00305-0

[41] Yozukmazl, A., Sunlu, F.S., Sunlu, U. and Ozsuer, M. (2011) The Determination of Organotin Compounds Levels in Sediment Samples from Turkish Aegean Sea Coast. Turkish Journal of Fisheries and Aquatic Sciences, 11, 649-660.

[42] Scrimshaw, M.D., Wahlen, R., Catterick, T. and Lester, J.N. (2005) Butyltin Compounds in a Sediment Core from the Old Tilbury Basin, London, UK. Marine Pollution Bulletin, 50, 1500-1507. http://dx.doi.org/10.1016/j.marpolbul.2005.06.018

[43] Üveges, M., Rodríguez-González, P., García Alonso, J.I., Sanz-Medel, A. and Fodor, P. (2007) Isotope Dilution Analysis Mass Spectrometry for the Routine Measurement of Butyltin Compounds in Marine Environmental and Biological Samples. Microchemical Journal, 85, 115-121. http://dx.doi.org/10.1016/j.microc.2006.04.003

[44] Gomez-Ariza, J.L., Beltrán, R., Morales, E., Giraldez, I. and Ruiz-Benitez, M. (1995) Acid Extraction Treatment of Sediment Samples for Organotin Speciation; Occurrence of Butyltin and Phenyltin Compounds on the Cadiz Coast, South-West Spain. Applied Organometallic Chemistry, 9, 51-64. http://dx.doi.org/10.1002/aoc.590090109

[45] Verslycke, T.A., Vethaak, A.D., Arijs, K. and Janssen, C.R. (2005) Flame Retardants, Surfactants and Organotins in Sediment and Mysid Shrimp of the Scheldt Estuary (The Netherlands). Environmental Pollution, 136, 19-31. http://dx.doi.org/10.1016/j.envpol.2004.12.008

[46] Tolosa, I., Bayona, J.M., Albaigés, J., Merlini, L. and De Bertrand, N. (1992) Occurrence and Fate of Tributyl- and Triphenyltin Compounds in Western Mediterranean Coastal Enclosures. Environmental Toxicology and Chemistry, 11, 145-155. http://dx.doi.org/10.1002/etc.5620110203 
[47] Shreadah, M.A., Said, T.O., Abd El Ghani, S.A. and Ahmed, A.M. (2011) Distribution of Different Organotin and Organolead Compounds in Sediment of the Gulf of Suez. Journal of Environmental Protection, 2, 545-554. http://dx.doi.org/10.4236/jep.2011.25063 
Scientific Research Publishing (SCIRP) is one of the largest Open Access journal publishers. It is currently publishing more than 200 open access, online, peer-reviewed journals covering a wide range of academic disciplines. SCIRP serves the worldwide academic communities and contributes to the progress and application of science with its publication.

Other selected journals from SCIRP are listed as below. Submit your manuscript to us via either submit@scirp.org or Online Submission Portal.
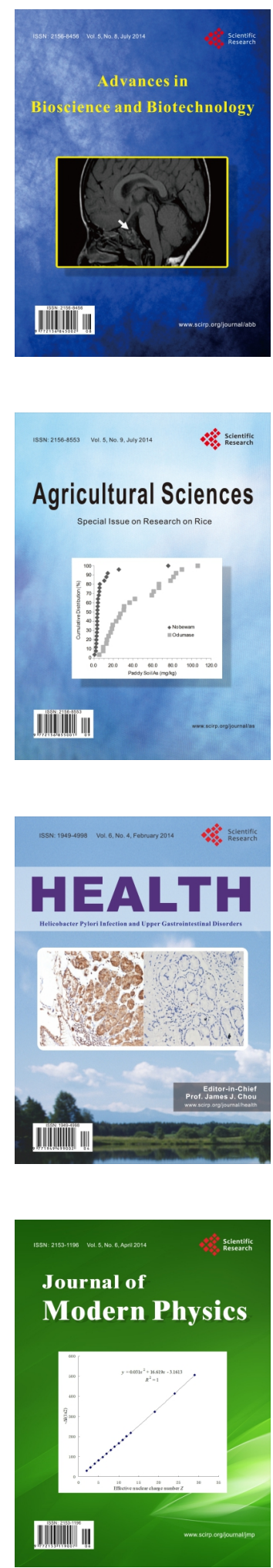
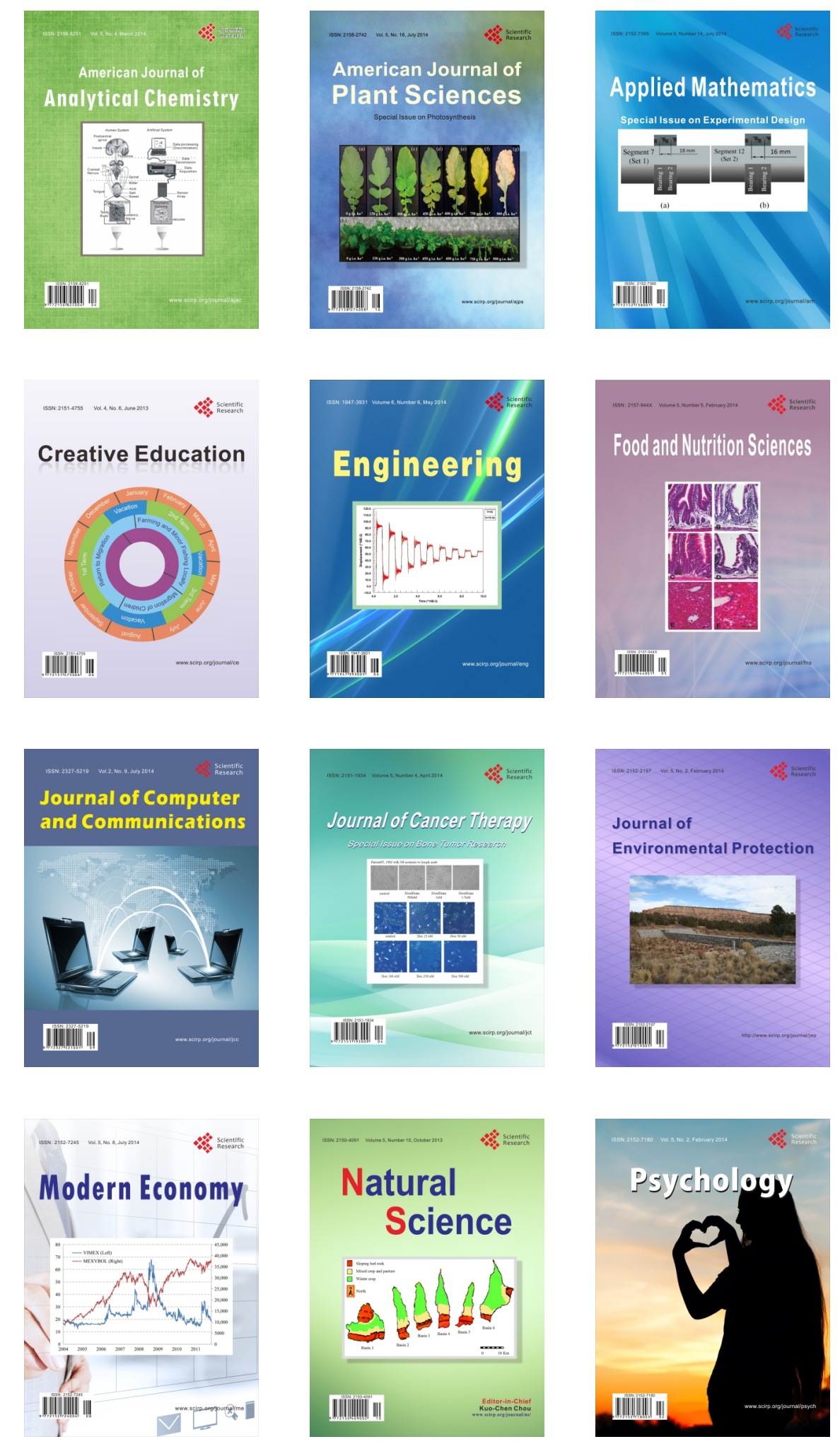